\title{
The value of hounsfield unit in retrograde intrarenal surgery versus percutaneous nephrolithotomy for the treatment of renal stone of 2-3 cm: A single-center prospective pilot study
}

\author{
Yuzhuo Li ${ }^{\text {a,\# }}$, Qiqi He ${ }^{\text {a,\#, }}$, Fei wang ${ }^{b}$, Teng Ma ${ }^{a}$, Junsheng Bao ${ }^{a}$, Li Yang ${ }^{a}$, Zhiping Wang ${ }^{a}$, Gupta \\ Sanjay ${ }^{\mathrm{c}}$
}

${ }^{a}$ Department of Urology, Key laboratory of disease of Urological systems, Gansu Nepho-Urological clinical Center, Second hospital of Lanzhou University, Lanzhou, Gansu, China.

${ }^{b}$ Department of Pediatrics, Second hospital of Lanzhou University, Lanzhou, Gansu, China.

${ }^{c}$ Department of Urology, University Hospitals Case Medicine Center, Case Western Reserve University, Cleveland, $\mathrm{OH}, \mathrm{USA}$.

\begin{abstract}
Background: To determine whether the Hounsfield Unit (HU) value of no-contrast computer computed tomography (NCCT) might offer better guidance in the selection of RIRS or PCNL in renal stones of 2-3 cm.

Methods: A total of 158 patients with kidney stones $(2-3 \mathrm{~cm}$ ) who underwent PCNL/RIRS from March 2016 to January 2019 were enrolled in this study. Age, gender, stone sizes, locations, average HU value of stones, surgery time, hospital stay time, stone-free rate, and complications at the time of hospitalization and 3-month follow-up were measured to identify the surgery efficiency.

Results: Upon consideration of the HU value, the mean surgery time was significantly decreased in RIRS comparing to the control RIRS (cRIRS) group (47.73 \pm 15.52 vs. $72.41 \pm 27.71 \mathrm{~min}, P<0.05)$. Statistically, the surgery time was strongly influenced by the HU values both in RIRS (OR 93.8, $P<0.01$ ) and PCNL (OR 8.21, $P<$ $0.05)$. HU values proved to have a strong positive relation with surgery time in RIRS while a low positive relation in PCNL $(P<0.05)$.

Conclusion: Overall, for renal stones of $2-3 \mathrm{~cm}$, RIRS might be a safe and efficacious treatment option if the HU value and other parameters could be comprehensively accounted for. Individual precision surgery might provide ideal treatment and prognosis for patients requiring long-term continuous clinical procedures.

Keywords: Hounsfield unit; retrograde intrarenal surgery; percutaneous nephrolithotomy; renal stones
\end{abstract}

\section{INTRODUCTION}

Renal stone disease is proved as one of the most frequent diseases in the urology clinical practice world-

\footnotetext{
\# These two authors contribute equally to the work as co-first authors.

* Corresponding author: Qiqi He

Mailing address: Department of Urology, Key laboratory of disease of Urological systems, Gansu Nepho-Urological clinical Center, Lanzhou University Second Hospital, 80 Cui Yinmen street, Lanzhou, Gansu, 730030, China.

E-mail: ery_heqq@izu.edu.cn

Received: 24 June 2021 / Accepted: 19 July 2021
}

wide. Extracorporeal shock wave lithotripsy (ESWL) has been introduced as an efficient alternative approach to disintegrate stones $<1 \mathrm{~cm}$. However, for the large stone with Hounsfield Unit (HU) $>1000$, the efficiency of this intervene is low ${ }^{[1]}$. Percutaneous nephrolithotomy (PCNL) has been increasingly utilized in the treatment of large stones nearing $80 \%$ success rates ${ }^{[2]}$. Nowadays, retrograde intrarenal surgery (RIRS) has been efficiently used in the clinic ${ }^{[3-5]}$. However, some stone diseases in the borderline are open to several surgery options determined by the size, stone burden, locations, etc., are not been all well standardized yet ${ }^{[4]}$. For instance, for $2-3 \mathrm{~cm}$ kidney stones, 
PCNL or RIRS with auxiliary ESWL are both feasible. However, PCNL might cause invasive complications and even lead to a slow loss of renal function in long run, while the RIRS could not resolve the problems in a single session, and a repeat session might raise the risk of ureteral stricture and other complications. Therefore, further individual precision surgery design is necessary.

The HU value calculated by non-contrast computed tomography (NCCT) has usually been underestimated in surgery procedures ${ }^{[6]}$. This perspective and singlecenter pilot study was therefore conducted to figure whether the HU value could be used as a decision aid in guiding RIRS and PCNL as an optimal procedure for patients with $2-3 \mathrm{~cm}$ renal stones.

\section{PATIENTS AND METHODS}

\section{Study population}

A prospective, double-blind study was undertaken from March 2016 to January 2019, which enrolled 98 patients and 60 control patients with renal calculi of 2-3 cm undergoing RIRS or PCNL. The study protocol was approved by the ethics committee of Lanzhou University Second Hospital (2018A-019). The informed consent was fully explained and signed by all patients.

\section{Study design and surgical techniques}

Inclusion criteria were the presence of renal stones 2-3 cm in diameter for all patients, surgery decisions (without evaluating the $\mathrm{HU}$ value) were initially evaluated and made by more than 20 years urology experienced chief doctors (ZP, Wang) based on the guideline and center clinical discussion ${ }^{[7]}$. All surgery was performed or supervised by another experienced urolithiasis surgeon (JS, Bao). After surgery, cases of $\mathrm{HU}<$ 1000 and performed RIRS were enrolled into the RIRS group, $\mathrm{HU} \geq 1000$ and performed PCNL were enrolled into the PCNL group. Meantime, randomization of two control groups (cRIRS and cPCNL) was determined by the sequences of patients' discharge time and surgery options. The appropriate cases that met the criterion were double-blind sorted into certain groups. Exclusion criteria of all cases were uncontrolled coagulopathy, anatomic abnormality, solitary kidney, pregnancy, and some challenge cases that might affect prognosis (huge BMI, long lower pole calyx, steep infundibularpelvic angle, and narrow infundibulum). Hemoglobin, platelet, coagulation tests, serum creatinine levels, and urine culture tests of patients were performed preoperatively, and treatment was provided according to the findings if necessary. Information was recorded on the patients' age, gender, HU value, stone sizes, and stone locations. The patients' enrollment algorithm is illustrated in Figure 1. The patients' data and groups' settings were gathered and developed by a resident (YZ, Li). The preoperative consultation and follow-ups were performed by another trained surgeon (T, Ma). The detailed surgery procedures were as follows.

\section{RIRS}

After general anesthesia, a safety guidewire was placed using rigid ureteroscopy (9.5/11.5 F), examined the ureter and possible pathologies as well as facilitate the placement of the ureteral access sheath. Stones were fragmented using a 270-micrometer laser fiber (Lumenis, San Jose, CA) with the help of a $7.5 \mathrm{~F}$ fiber optic flexible ureterorenoscope (Storz FLEX-X2, Tuttlingen, Germany) after the placement of ureteral access sheaths (9.5/11.5 F) (COOK, Indiana, USA). Stone fragmentation was then dusting accomplished using laser energy of 0.8-1.2 J and a rate of $10-25 \mathrm{~Hz}$ (Lumenis, San Jose, CA), which was adjusted based on stone situations. Stones smaller than $2 \mathrm{~mm}$ were left to pass spontaneously while the larger ones were removed with a basket catheter. A 4.8 F Double-J (D-J) stent was routinely placed at the end of the operation because of possible edema and other problems that might be caused by the access sheath. For the patients who could not place the access sheaths rightly due to the small diameter of their ureters, and a D-J stent was placed for 2-4 weeks and then perform the surgery ${ }^{[8,9]}$. PCNL

Solo ultrasonography- (US-) guided PCNL were performed on the patients considered not fit for RIRS. Solo US-guided PCNL technique was described previously ${ }^{[10,11]}$. After general anesthesia was accomplished, an externalized SF ureteral catheter was placed into the ureter via a cystoscope and dilate the kidney by retrograde instillation of saline if needed. A prone position was then performed. We used a $3.5-\mathrm{MHz}$ convex abdominal transducer (Hitachi Aloka Medical America, Wallingford, CT) to localize the stone position as well as the collecting system anatomy. The renal puncture was performed with an 18-gauge EchoTip needle (Cook Medical) under real-time ultrasonographic monitoring freehand without the aid of a needle guide. A safety guidewire was placed into aim calyx to confirm the working tract via needle. An $10 \mathrm{~F}$ to $18-20 \mathrm{~F}$ dilated sheath (BARD X-Force, Bard Medical) was used for working tract dilation via the guidewire. Thus, the lith- 
otripter device could be used for stone fragmentation and removal via working tract ( $18 \mathrm{~F}$ or $20 \mathrm{~F}$ ). The $5 \mathrm{~F}$ externalized ureteral catheter was removed after stone dusting, an 5F D-J tube stent, an $12 \mathrm{~F}$ catheter, and an 16-18F drainage tube (depends on working tract diameter) were indwelling in the ureter (4 weeks), urethral (3 days) and renal calyx ( 1 week), respectively.

\section{Outcome assessment}

The primary outcome was the stone-free rate (SFR). The final SFR was determined by NCCT taken in the third postoperative month. The procedure was considered successful if there were no residual stones.

The secondary outcomes included the relationships between SFR and stone density (HU value), locations, hospitalization time, operative time, also complications. Surgical times were calculated from the beginning of cystoscopy to the end of the procedure carried out by placing a urinary catheter. The mean length of hospital stay was calculated from the day of surgery to the day of discharge. The Clavien classification was used for the postoperative and follow-up complications assessment.

\section{Statistical analysis}

Data obtained from this study were analyzed using the SPSS 20.0 statistics software. While investigating whether the variables were normally distributed, Shapiro Wilk's was used due to the number of units. The Mann-Whitney $U$ test was used for analyzing the differences between the groups due to the non-normal distribution of the variables. The relationship between the categorical variables was analyzed by Chi-square or Fisher exact tests. Regression analysis was used to evaluate the correlation of the data. The HU value was considered as a dependent variable whereas all other significant potential parameters were taken as independent variables. Univariate and multivariate analysis was performed to obtain the odds ratio, 95\% confidence interval, and $P$-value. The results were interpreted using a significance level of 0.05 .

\section{RESULTS}

\section{Patients and stone characteristics}

The study was completed with a total of 158 patients, divided into 4 groups: RIRS group $(n=62)$, PCNL group ( $n=36)$, control RIRS group (cRIRS, $n=30$ ), and control PCNL group (cPCNL, $n=30$ ). The preoperative characteristics of the patients and the stones are shown in Table 1.
When we set a paired match between surgery and control groups, the age, stone sizes did not show a significant difference between RIRS and cRIRS groups $(P>0.05)$. The PCNL and $c P C N L$ groups were similar in terms of these aspects $(P>0.05)$; however, the HU value of the RIRS group was significantly lower than the value of the cRIRS group $(P<0.05)$. When we set a match of the RIRS and PCNL groups, the PCNL stone sizes were significantly larger than RIRS, more upper and middle stones were treated by RIRS while lower pole stones and multiple stones were treated by PCNL $(P<0.05)$. The HU value of the PCNL group was higher than that of the RIRS group $(P<0.05)$ (details showed in Table 1).

\section{The outcomes and follow-ups}

In terms of mean surgery time, the RIRS group demonstrated a shorter surgery time than cRIRS. While the PCNL patients need to puncture solo holes to perform the surgery and retrograde ureteral catheterization before the procedure, which might take more time than the RIRS group $(P<0.05)$. In all RIRS and cRIRS group patients ( $n=92), 18$ patients suffered temporary fever after surgery, a slight abdominal or flank pain/blood urine was recorded as Clavien grade 1 complication and was overcome by symptomatic treatment (alternating antibiotics, antipyretics, etc.). None of these two groups suffered Clavien grade 2-3 complications in short-term post-operation. However, the PCNL and cPCNL groups exhibited more complications than RIRS post-operation $(P<0.05)$. A total of 38 out of 66 patients who performed PCNL exhibited postoperative symptoms. The 34 patients were recorded as Clavien grade 1 complication (17 in each PCNL group), 2 patients (each in two PCNL groups) had to receive the blood transfusion as a result of the hemorrhage of the renal cortex, and a second session PCNL was performed after 2 weeks, which was recorded as Clavien grade 2 and 3 complications. For mean hospital stay time, patients in the RIRS group had a shorter duration compared with the PCNL group as the less severe postoperative reactions and complications. No differences were found between RIRS vs. cRIRS or PCNL vs. cPCNL in Table 2.

Three months post-operation, the SFR of PCNL groups demonstrated a significant elevation compared to RIRS $(P<0.05)$. However, no significant differences were found in the intergroup comparison of RIRS vs. cRIRS and PCNL vs. cPCNL. Two patients of the cRIRS group were readmitted after 3 months post-operation, one 


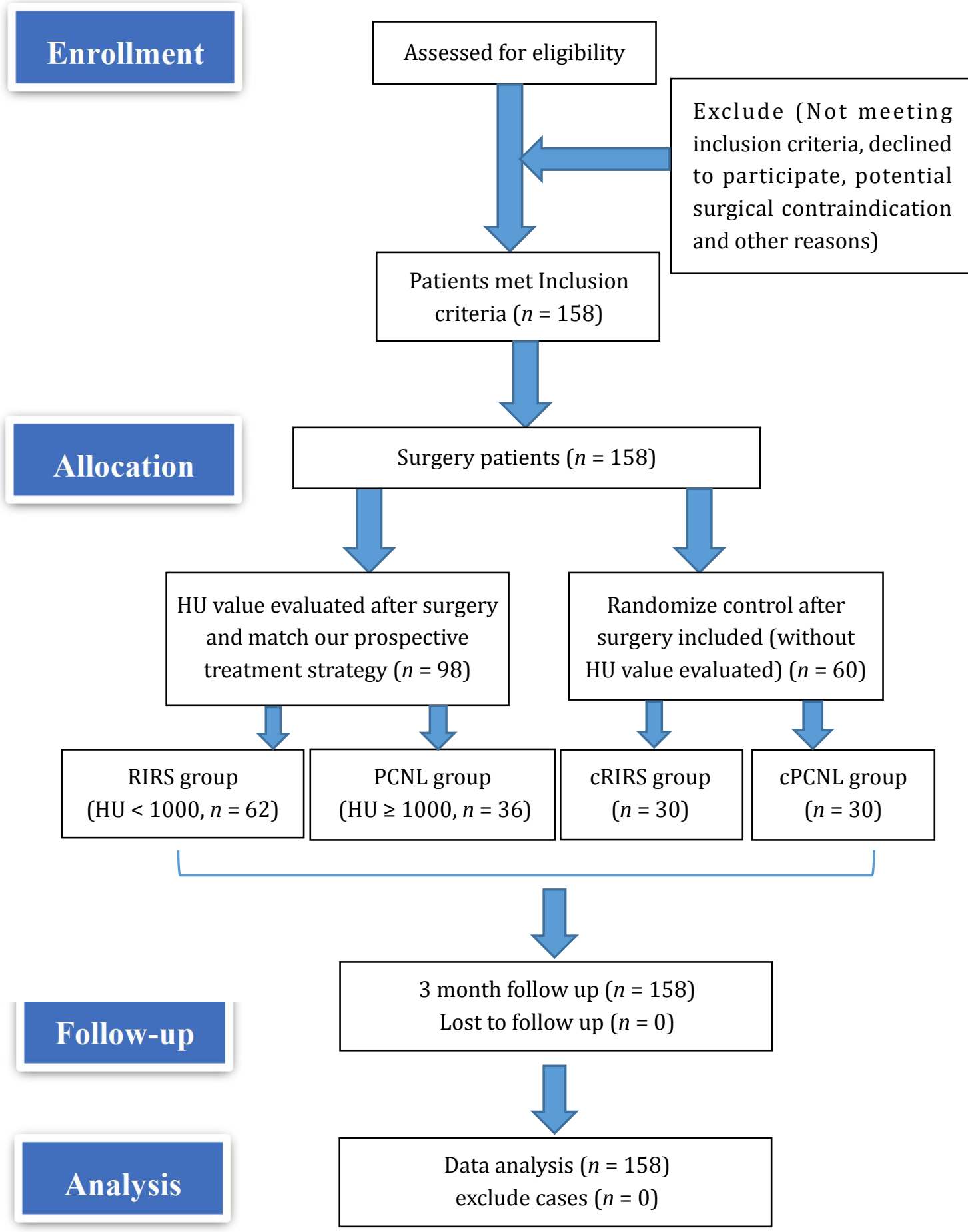

Figure 1. Patients' enrollment algorithm.

of them appeared ureter stone-street treated by ESWL failed, then by rigid ureteroscopic lithotripsy, and the other patient suffered from continuous fever 4 months post-operation, an ultrasonic guild nephrostomy was performed and second session PCNL was performed 2 weeks later, then recovered and discharged 6 days postop. No Clavien grade 1-2 complications were noted in the 3-month follow-up, the ESWL was enrolled as adjuvant therapy for all patients of residual stones (Table 2). Factor analysis of HU value towards total outcomes After the evaluations mentioned above, to explore the role of the HU value predicting outcomes, univariate and multivariate analyses were carried out for a thresh- 


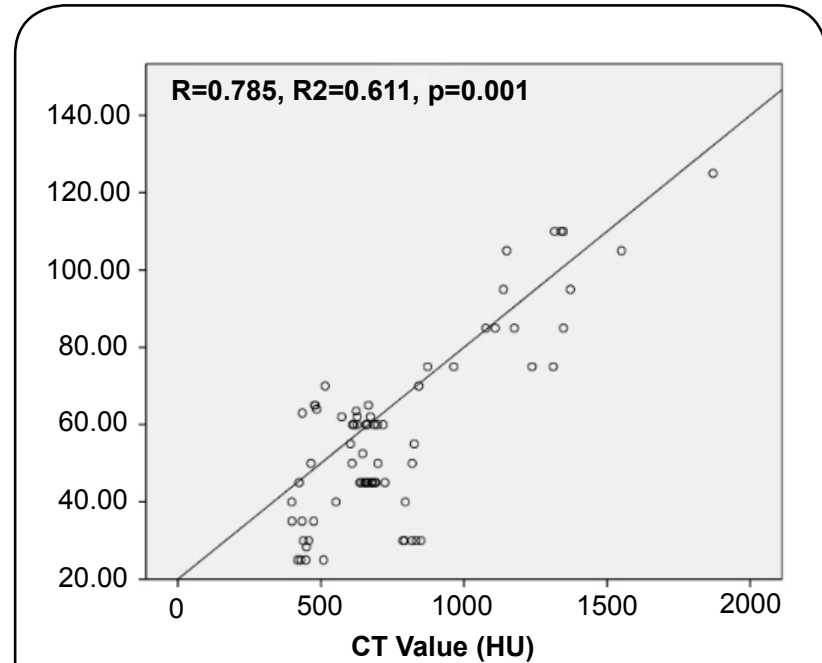

Figure 2. Correlation analysis between the HU values to surgery time in RIRS.

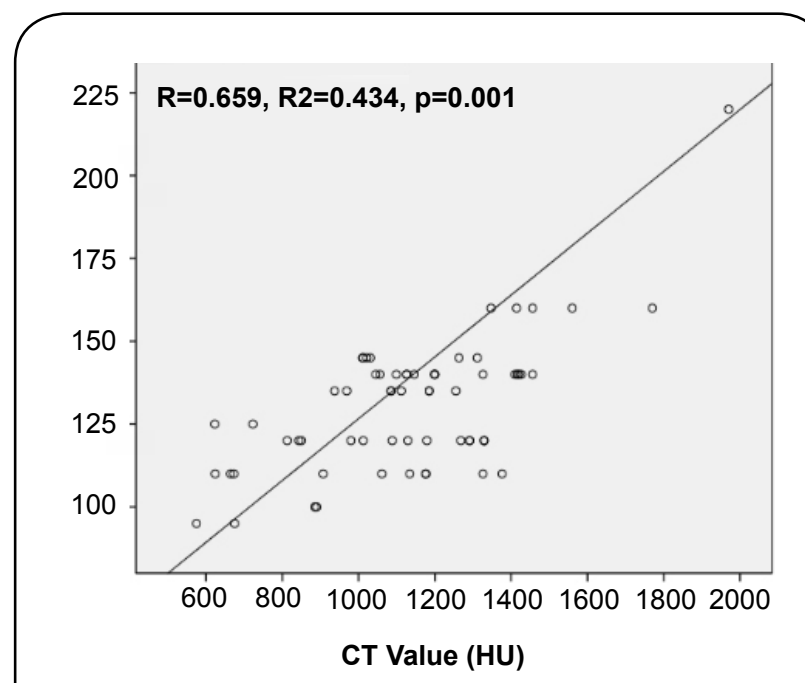

Figure 3. Correlation analysis between the HU values and surgery time in PCNL.

old of $1000 \mathrm{HU}$ on all 158 cases. Considering different procedures and difficulties in respect, RIRS and PCNL were analyzed respectively. It proved that only surgery time might be strongly influenced by the HU values both in RIRS (OR 93.8, $P<0.01$ ) and PCNL (OR 8.21, $P$ $<0.05)$. No significant differences were noted in terms of the SFR, hospital stay, and complications $(P>0.05)$ (Table 3).

\section{Correlation analysis}

Correlation analysis revealed that the HU values were significant and positively related to the surgery time if RIRS were adopted (Figure 2), whereas slight positive if PCNL was applied (Figure 3). However, other outcome indexes did not prove a significant relation to the $\mathrm{HU}$ values (data not shown).

\section{DISCUSSION}

PCNL has been recommended as the first-line treatment for intrarenal stones larger than $2 \mathrm{~cm}$ according to the European Association of Urology Guidelines ${ }^{[12]}$. Although the advantage of high stone clearance rates has been widely proved in reports, the invasive method with serious complications might also worry urologists inevitably. At the same time, RIRS has been recommended as the first-line treatment targeting intrarenal stones between 1-2 cm, in particular, for patients of obesity, anticoagulation, skeletal deformity, and renal anomalies, the indications of RIRS could be broadened ${ }^{[12-15]}$.

Several studies have already attempted to employ the RIRS to treat the stone $>2 \mathrm{~cm}$ and exhibiting some positive effects. However, despite these positive results, RIRS is still considered escalating the treatment cost and requires multiple sessions to clear large stone $>2 \mathrm{~cm}$ in the current strategy. Long surgery time and several sessions also might lead to serious complications such as stricture and fibrosis of the ureter ${ }^{[16]}$, it has been proved that the elevated levels of renal injury biomarker increase further according to stone size and surgery time, which means larger stone and longer surgery time would raise the risk of renal injury positively ${ }^{[17,18]}$, whereas PCNL provides an SFR around 95\% but with potential invasive complications after the first treatment ${ }^{[19-21]}$. The optimal surgery decisions for 2-3 cm stone become a topic worth pondering and need to be elucidated.

The HU value of NNCT for kidney stones is usually used for ESWL preparation. The stone density has been reported to relate to ESWL outcome. Stones $\geq$ $1000 \mathrm{HU}$ are less likely to be disintegrated ${ }^{[22,23]}$. However, urologists usually focus on this indicator in ESWL but are likely to neglect it before lithotripsy. No studies focused on the outcomes of large stones $(>2 \mathrm{~cm})$ performed by RIRS with the HU value evaluated so far to our knowledge. In our clinical experiences, the stone density $<1000$ HU could be easily fragmenting initially and then dusting to suitable size particles by adjusting holmium laser settings using RIRS, except the stone of low calyx or severe hydronephrosis, which might be more suitable to perform PCNL or mPCNL. For the stones > $1000 \mathrm{HU}$, some cases might need a long time and are not easy to dusting. Some studies confirmed our experiences and presented that the lower efficiency of holmium laser in some stone cases ${ }^{[24]}$. Moreover, lasers in the undeveloped area might not work well 
in all stones, especially for large stones, which need a long surgery time and easily cause complications. Even with a high-tech laser, the residual stone could not be avoided in all cases probably. Due to existed controversy and limited literature, we reasoned that it would be meaningful to conduct this small prospective singlecenter pilot studies to define the potential decision aid of screening the $\mathrm{HU}$ value on $2-3 \mathrm{~cm}$ renal calculus to perform RIRS or PCNL and following outcomes.

The highlight of our present study is that we firstly proposed that the HU value should be taken as a routine consideration in 2-3 cm stone diseases requiring RIRS or PCNL surgery which might change the surgery decision in some cases. The threshold of $1000 \mathrm{HU}$ is widely accepted in the ESWL procedure. It might not be accurate but effective in the preparation of RIRS. Larger renal stones need a significantly longer operation time, which essentially increased the risk of sepsis, especially in RIRS. Without prompt management, sepsis would be dangerous and even life-threatening ${ }^{[25]}$. Stiff stones $>2 \mathrm{~cm}$ with high density might not fit for RIRS and could be easily performed by PCNL. Fragile stones (postulate $\mathrm{HU}<1000$ ) might be the optimal candidate for RIRS and might benefit patients. Adjuvant ESWL would be also meaningful and efficacious to the residual stones less than $1000 \mathrm{HU}$ if necessary. The second session RIRS or PCNL might be omitted. During our preliminary study, some cases of easily disintegrated stones were noticed and marked, which mostly proved $\mathrm{HU}<1000$, which is consistent with some literature results ${ }^{[1,2]}$. Therefore, we organized 4 groups to assess if $1000 \mathrm{HU}$ might be useful in RIRS or PCNL. The results exhibited that with consideration of $\mathrm{HU}$ value, no severe complications and repeat sessions were noted in the RIRS group and adjuvant ESWL was all efficient. The surgery time, hospital stay, and complications of RIRS after HU value evaluated were superior to cRIRS and two PCNL groups.

Our results confirmed that PCNL offered a higher SFR than RIRS as well, which was consistent with most published studies ${ }^{[2,9,21]}$. Zewu et al. supposed that the potential reasons for RIRS with a relatively lower SFR may be due to residual fragments that are more likely to represent a cluster of clinically insignificant fragments and small working channel according to literature ${ }^{[26]}$, as well as a call for the vacuum aspiration stone system for RIRS. However, RIRS with the HU value evaluated could provide a comparable final SFR and shorter recovery time with fewer overall complications for intermediate-size renal stones $(2-3 \mathrm{~cm})$ in one session with help of adjuvant ESWL in many cases. During the follow-ups, best overcomes were raised from patients of RIRS with HU value evaluated, none received a second session RIRS and ureteral stricture or other severe complications.

\section{LIMITATIONS AND RECOMMENDATIONS}

Several limitations need to be taken into account when considering the present work. Firstly, the timing of the outcome evaluation and follow-up might not be long enough. Some residual stone fragments may pass away in 6-12 months after surgery, the 3 months' endpoint seems not enough and may overestimate our failure rate ${ }^{[27]}$. Secondly, among the present patients, all RIRS groups with a stone density of $<1000 \mathrm{HU}$ and had no complications in the 3-month follow-up. This result indicated that the $1000 \mathrm{HU}$ value played a critical role in predicting the success of RIRS and exclude those who might suffer a poor outcome or complications. The fact we need to know is that these patients initially decided to perform RIRS properly and then following set groups by the HU value were evaluated. Some cases of the cRIRS group were still $>1000 \mathrm{HU}$ and proved that RIRS remained efficacious management. The threshold, comprehensive indicators, and network approach may need further investigation. The third limitation is the consistency of stone fragility and the HU value. For most cases, the $\mathrm{HU}$ value could offer predictive and corresponding information before surgery. However, pure cystine stones with $\mathrm{HU}<1000$ and account for less than $2 \%$ of all cases of lithiasis might not be easily fragmented and dusted by employing RIRS if stones are larger than $2 \mathrm{~cm}$. Therefore, for the small group of patients with family calculus history, young age, and metabolic abnormality, more attention before surgery is needed. Lastly but foremost, participant size and study design are limited, we tried to identify and enroll as many patients into this study to draw more accurate results. However, it might not be easy for a single center to conduct an ideal participants' size of particular range stones $(2-3 \mathrm{~cm})$ for analysis though the total number of stone surgery at our center was high $(>800$ cases/year). Given the large sample numbers and long follow-up periods, it seems likely study of this magnitude might probably miss follow-up data and generate bias. Only control cases of our study were randomized, so it could not avoid the potential bias similar to an observational study, and could not raise some evidence as strong as a standard random control trial (RCT). The conclusions should be careful considered upon these 
Table 1. Patients and Stone Characteristics.

\begin{tabular}{|c|c|c|c|c|c|c|}
\hline & $\operatorname{RIRS}(n=62)$ & cRIRS $(n=30)$ & $P$ - value ${ }^{b}$ & PCNL $(n=36)$ & cPCNL $(n=30)$ & $P$ - value ${ }^{\mathrm{b}}$ \\
\hline \multirow[t]{2}{*}{ Gander [n (\%)] } & M: 48 (77.4) & M: $18(60.0)$ & 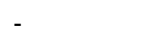 & M: 27 (75.0) & M: 23 (76.7) & - \\
\hline & F: $14(22.6)$ & $F: 12(40.0)$ & & F: 9 (25.0) & F: 7 (23.3) & \\
\hline Age (Y.O) & $46.82 \pm 12.23$ & $49.07 \pm 10.68$ & 0.640 & $45.94 \pm 9.99$ & $44.75 \pm 12.71$ & 0.100 \\
\hline Stone size $(\mathrm{cm})$ & $2.43 \pm 0.42$ & $2.27 \pm 0.36$ & 0.710 & $2.70 \pm 0.29^{\mathrm{a}}$ & $2.73 \pm 0.32$ & 0.471 \\
\hline \multicolumn{7}{|c|}{ Main stone Location [n (\%)] } \\
\hline Up/middle pole & $44(70.96)$ & $16(53.33)$ & 0.150 & $7(19.44)^{a}$ & $3(10.00)$ & 0.470 \\
\hline Pelvis & $16(25.81)$ & $11(36.67)$ & 0.407 & $13(36.11)$ & $19(63.33)$ & 0.051 \\
\hline Low pole & $2(3.22)$ & $3(10.00)$ & 0.393 & $16(53.33)^{\mathrm{a}}$ & $8(26.57)$ & 0.505 \\
\hline Multiple stone [n (\%)] & $4(6.45)$ & $6(20.00)$ & 0.109 & $10(27.78)^{a}$ & $7(23.33)$ & 0.897 \\
\hline CT value (HU) & $628.78 \pm 145.57$ & $994.13 \pm 387.34$ & $<0.001$ & $1254.17 \pm 169.70^{\mathrm{a}}$ & $989.55 \pm 297.63$ & 0.352 \\
\hline
\end{tabular}

${ }^{\mathrm{a}}$ Variables were compared by independent samples t-test or Chi-square/Fisher exact tests (RIRS vs. PCNL, $P<0.05$ ). ${ }^{\mathrm{b}}$ Variables were compared by independent samples t-test or Chi-square/Fisher exact tests (RIRS vs. cRIRS, PCNL vs. cPCNL). Data are presented as mean \pm SD or number (\%).

Table 2. Operative and postoperative data of the RIRS and PCNL groups and their comparisons.

\begin{tabular}{|c|c|c|c|c|c|c|}
\hline & $\operatorname{RIRS}(n=62)$ & cRIRS $(n=30)$ & $P$ - value ${ }^{\mathrm{b}}$ & PCNL $(n=36)$ & cPCNL $(n=30)$ & $P$ - value ${ }^{b}$ \\
\hline Surgery time (min) & $50.47 \pm 18.04$ & $72.28 \pm 28.42$ & 0.003 & $134.44 \pm 14.62^{\mathrm{a}}$ & $125.86 \pm 24.38$ & 0.124 \\
\hline Hospital stays (days) & $3.31 \pm 1.70$ & $3.55 \pm 1.52$ & 0.913 & $5.01 \pm 2.02^{\mathrm{a}}$ & $5.41 \pm 2.45$ & 0.170 \\
\hline \multicolumn{7}{|c|}{ Complications (post-op) } \\
\hline CG $1(\%)$ & $11(17.74)$ & $7(23.33)$ & 0.723 & $17(47.22)^{\mathrm{a}}$ & $17(56.67)$ & 0.605 \\
\hline CG $2(\%)$ & $0(0)$ & $0(0)$ & - & $1(2.7)$ & $1(3.33)$ & 0.555 \\
\hline CG $3(\%)$ & $0(0)$ & $0(0)$ & - & $1(2.7)$ & $1(3.33)$ & 0.555 \\
\hline 3-month SFR [n (\%)] & $48(77.42)$ & $20(66.67)$ & 0.396 & $34(94.44)^{a}$ & $29(96.67)$ & 0.871 \\
\hline \multicolumn{7}{|c|}{ Complications (3 month) } \\
\hline CG $1(\%)$ & $0(0)$ & $0(0)$ & - & $6(16.67)^{\mathrm{a}}$ & $3(10.00)$ & 0.670 \\
\hline CG $2(\%)$ & $0(0)$ & $0(0)$ & - & $0(0)$ & $0(0)$ & - \\
\hline CG $3(\%)$ & $0(0)$ & $2(6.67)$ & 0.196 & $0(0)$ & $0(0)$ & - \\
\hline
\end{tabular}

CG: Clavien grade, post-op: post-operation. ${ }^{a}$ Variables were compared by independent samples t-test or Chi-square/Fisher exact tests (RIRS

Table 3. Factor analysis of surgery outcomes on the threshold of density at $1000 \mathrm{HU}$.

\begin{tabular}{|c|c|c|c|c|c|}
\hline & & \multicolumn{2}{|c|}{ Univariate } & \multicolumn{2}{|c|}{ Multivariate } \\
\hline & & $\begin{array}{l}\text { Odds ratio } \\
\text { (95\% Confidence } \\
\text { interval) }\end{array}$ & $P$-value & $\begin{array}{l}\text { Odds ratio } \\
\text { (95\% Confidence } \\
\text { interval) }\end{array}$ & $P$-value \\
\hline \multirow{4}{*}{$\operatorname{RIRS}(n=92)$} & Stone free rate & $1.53(0.46,5.03)$ & 0.705 & - & - \\
\hline & Surgery time $<60 \mathrm{~min}$ & $93.8(11.09,793.11)$ & $<0.001$ & $35.1(9.25,391.62)$ & $<0.001$ \\
\hline & Hospital stay $<4$ days & $1.33(0.41,4.36)$ & 0.423 & - & - \\
\hline & Complications & $0.67(0.19,2.38)$ & 0.373 & - & - \\
\hline \multirow{4}{*}{ PCNL $(n=66)$} & Stone free rate & $0.63(0.05,7.39)$ & 0.572 & - & - \\
\hline & Surgery time $<130 \mathrm{~min}$ & $8.21(1.69,39.99)$ & 0.003 & $3.59(1.36,28.56)$ & 0.125 \\
\hline & Hospital stay $<5$ days & $1.05(0.29,3.95)$ & 0.608 & - & - \\
\hline & Complications & $0.66(0.21,2.06)$ & 0.335 & - & - \\
\hline
\end{tabular}


limited contributions. Therefore, a large sample size and multi-center study remain to call for.

After all, it is important to balance benefits and risks according to the characteristics of individuals and select an ideal treatment for patients. Tubeless SMP might be a great choice for these cases of middle/lower pole stones with $\mathrm{HU}>1000$ nowadays but remain need to be popularized. However, our study further indicated that RIRS might be a safe and efficacious treatment option for renal stones of $2-3 \mathrm{~cm}$ in size if the HU value has been considered for evaluation. Many patients thus might not have to suffer the potentially severe complications of PCNL. Although both the EAU and AUA guidelines do not currently recommend RIRS as the first-line treatment of $2-3 \mathrm{~cm}$ stones, we indeed have the confidence to utilize it as a primary modality if patients are properly selected. Nevertheless, further prospective randomized multi-center trials are required to confirm these results.

\section{CONCLUSION}

RIRS might be a safe and efficacious treatment option for renal stones of $2-3 \mathrm{~cm}$ if HU value $<1000$. Take the sight of HU value before RIRS in $2-3 \mathrm{~cm}$ kidney stone cases, several sessions of RIRS might be prevented and decrease the risk of complications. Some patients might receive better outcomes only in a single session of RIRS if patients could be properly selected and well prepared. Individual precision surgery might provide ideal treatment and prognosis for patients requiring long-term continuous clinical procedures.

\section{DECLARATIONS}

\section{Authors' contributions}

Yuzhuo Li: Paper writing, collected the data.

Qiqi He: Paper writing, analysis the final data, study design.

Fei Wang and Teng Ma: Collected the data.

Junsheng Bao and Li Yang: Surgery performances.

Zhiping Wang: Surgery method design.

Sanjay Gupta: Analysis the final data, review and revise the paper.

\section{Acknowledgment}

The authors thank all the medical stuffs who take part into this work.

Funding

This work was supported by the National Natural Science Foundation of China (81800671), the Industry
Program of Gansu and Cuiyin Talent Program (CYQN06).

\section{Conflict of interest}

Qiqi He is a member of the Editorial Board of Clinical Surgery Research Communications and other authors declare no conflict of interest. All authors were not involved in the journal's review or desicions related to this manuscript.

\section{REFERENCES}

1. M. M, A., M. A, A., A. A.-D, A., S. M, A., S. Z, A., \& O, $M .(2014)$. The success of extracorporeal shockwave lithotripsy based on the stone-attenuation value from non-contrast computed tomography. Arab Journal of Urology, 12(2), 155-161.

2. Bai, Y., Wang, X., Yang, Y., Han, P., \& Wang, J. (2017). Percutaneous nephrolithotomy versus retrograde intrarenal surgery for the treatment of kidney stones up to $2 \mathrm{~cm}$ in patients with solitary kidney: a single centre experience. Bmc Urology, 17(1), 1-6.

3. Kim, T. J., Lee, I. J., Lee, J. K., Lee, H. M., Jeong, C. W., Hong, S. K., ... \& Oh, J. J. (2018). Analysis of factors affecting re-admission after retrograde intrarenal surgery for renal stone. World Journal of Urology, 37(6), 1205-1210.

4. Sabnis, R. B., Ganesamoni, R., Doshi, A., Ganpule, A. P., Jagtap, J., \& Desai, M. R. (2013). Micropercutaneous nephrolithotomy (microperc) vs retrograde intrarenal surgery for the management of small renal calculi: a randomized controlled trial. Journal of Urology, 112(3), 355-361.

5. Aydemir, H., Budak, S., Kumsar, S., Kose, O., Saglam, H. S., \& Adsan, O. (2014). Efficacy of surgical techniques and factors affecting residual stone rate in the treatment of kidney stones. Turkish Journal of Urology, 40(3), 144-149.

6. Gok, A., Polat, H., Cift, A., Yucel, M. O., Gok, B., Sirik, M., ... \& Kalyenci, B. (2015). The hounsfield unit value calculated with the aid of non-contrast computed tomography and its effect on the outcome of percutaneous nephrolithotomy. Urolithiasis, 43(3), 277-281.

7. Campbell-Walsh. (11th ed) Urology. Philadelphia. Elsevie 2015.

8. Hamed, A. M. A. E., Elmoghazy, H., Aldahshoury, M., Riad, A., Mostafa, M., Farag, F., \& Gamal, W. (2017). Single session vs two sessions of flexible ureterosopy (FURS) for dusting of renal pelvic stones 2-3 $\mathrm{cm}$ in diameter: Does stone size or hardness play a role in number of sessions to be applied?". Turkish 
Journal of Urology, 43(2), 158-161.

9. Li, M. M., Yang, H. M., Liu, X. M., Qi, H. G., \& Weng, G. B. (2018). Retrograde intrarenal surgery vs miniaturized percutaneous nephrolithotomy to treat lower pole renal stones $1.5-2.5 \mathrm{~cm}$ in diameter. World Journal of Clinical Cases, 6(15), 931-935.

10. Usawachintachit, M., Tzou, D. T., Hu, W., Li, J., \& Chi, T. (2017). X-ray-free Ultrasound-guided Percutaneous Nephrolithotomy: How to Select the Right Patient? Urology, 100, 38-44.

11. Zhou, T., Chen, G., Gao, X., Zhang, W., Xu, C., Li, L., \& Sun, Y. (2015). 'X-ray'-free balloon dilation for totally ultrasound-guided percutaneous nephrolithotomy. Urolithiasis, 43(2), 189-195.

12. Türk, C., Petř́ik, A., Sarica, K., Seitz, C., Skolarikos, A., Straub, M., \& Knoll, T. (2016). EAU guidelines on interventional treatment for urolithiasis. European Urology, 69(3), 475-482.

13. Zanetti, S. P., Boeri, L., Catellani, M., Gallioli, A., Trinchieri, A., Sarica, K., \& Montanari, E. (2016). Retrograde intrarenal surgery (RIRS), regular and small sized percutaneous nephrolithotomy (PCNL) in daily practice: European Association of Urology Section of Urolithiasis (EULIS) Survey. Archivio Italiano di Urologia e Andrologia, 88(3), 212-216.

14. Ruhayel, Y., Tepeler, A., Dabestani, S., MacLennan, S., Petř́ik, A., Sarica, K., ... \& Türk, C. (2017). Tract sizes in miniaturized percutaneous nephrolithotomy: a systematic review from the european association of urology urolithiasis guidelines panel. European Urology, 72(2), 220-235.

15. Javanmard, B., Razaghi, M. R., Jafari, A. A., \& Mazloomfard, M. M. (2015). Flexible ureterorenoscopy versus extracorporeal shock wave lithotripsy for the treatment of renal pelvis stones of 10-20 $\mathrm{mm}$ in obese patients. Journal of lasers in medical sciences, 6(4), 162-166.

16. Dong, H., Peng, Y., Li, L., \& Gao, X. (2018). Prevention strategies for ureteral stricture following ureteroscopic lithotripsy. Asian Journal of Urology, 5(2), 94-100.

17. Balasar, M., Pişkin, M. M., Topcu, C., Demir, L. S., Gürbilek, M., Kandemir, A., \& Öztürk, A. (2016). Urinary kidney injury molecule-1 levels in renal stone patients. World journal of urology, 34(9), 13111316.

18. Dede, O., Dağguli, M., Utanğaç, M., Yuksel, H., Bodakcı, M. N., Hatipoğlu, N. K., ... \& Penbegül, N.(2015). Urinary expression of acute kidney injury biomarkers in patients after RIRS: it is a prospec-tive, controlled study. International Journal of Clinical \& Experimental Medicine, 8(5), 8147-8152.

19. Guo Jingyang. (2018). Clinical effects of PolyFlexible Ureteroscopy combined with ultrasound guided all seeing needle Microperc to treatment of Lower Pole Renal Calculi larger than $2 \mathrm{~cm}$ with infundibulopelvic angle less than 60 degrees. Journal of Current Medical Research and Opinion, 1(3).

20. Kucukdurmaz, F., Sahinkanat, T., Olmez, C., Temizer, M., \& Resim, S. (2018). Comparison of ureteroscopy and percutaneous nephrolithotomy for renal pelvic stones over $2 \mathrm{~cm}$. Journal of Clinical and Analytical Medicine, 9(1), 42-46.

21. Çakıcı, M. Ç., Sarı, S., Özok, H. U., Karakoyunlu, N., Hepşen, E., Sağnak, L., ... \& Ersoy, H. (2018). Comparison of Retrograde Intrarenal Surgery and Percutaneous Nephrolithotomy in the Treatment of 2-3 cm Multicalyceal Kidney Stones. Journal of Urological Surgery, 5(4), 143-148.

22. Waqas, M., Imran Jamil, M., Ayaz Khan, M., \& Akhter, S. (2018). Evaluating the importance of different computed tomography scan-based factors in predicting the outcome of extracorporeal shock wave lithotripsy for renal stones. Investigative and clinical urology, 59(1), 25-31.

23. Celik, S., Sefik, E., Basmacı, I., Bozkurt, I. H., Aydın, M. E., Yonguc, T., \& Degirmenci, T. (2018). Anovel method for prediction of stone composition: the average and difference of Hounsfield units and their cut-off values. International Urology and Nephrology, 50(8), 1397-1405.

24. Ito, H., Kawahara, T., Terao, H., Ogawa, T., Yao, M., Kubota, Y., \& Matsuzaki, J. (2012). Predictive value of attenuation coefficients measured as Hounsfield units on noncontrast computed tomography during flexible ureteroscopy with holmium laser lithotripsy: a single-center experience. Journal of Endourology, 26(9), 1125-1130.

25. Cindolo, L., Berardinelli, F., Castellan, P., Castellucci, R., Pellegrini, F., \& Schips, L. (2017). A fatal mycotic sepsis after retrograde intrarenal surgery: a case report and literature review. Urologia Journal, 84(2), 106-108.

26. Zewu, Z., Cui, Y., Feng, Z., Yang, L., \& Chen, H. (2019). Comparison of retrograde flexible ureteroscopy and percutaneous nephrolithotomy in treating intermediate-size renal stones $(2-3 \mathrm{~cm})$ : a metaanalysis and systematic review. International braz j urol, 45(1), 10-22. 
27. Ouzaid, I., Al-Qahtani, S., Dominique, S., Hupertan, V., Fernandez, P., Hermieu, J. F., ... \& Ravery, V. (2013). A 970 Hounsfield units (HU) threshold of kidney stone density on non-contrast computed tomography (NCCT) improves patients' selection for extracorporeal shockwave lithotripsy (ESWL): evidence from a prospective study. Bju International, 110(11b), E438-E442. 